\title{
Adaptive changes of human islets to an obesogenic environment in the mouse
}

\author{
S. Gargani • J. Thévenet • J. E. Yuan • B. Lefebvre • \\ N. Delalleau • V. Gmyr • T. Hubert • A. Duhamel • \\ F. Pattou • J. Kerr-Conte
}

Received: 31 May 2012/Accepted: 24 October 2012 / Published online: 29 November 2012

(C) The Author(s) 2012. This article is published with open access at Springerlink.com

\begin{abstract}
Aims/hypothesis In this study, we used an immunodeficient mouse model to explore, in vivo, the longitudinal adaptation of human islets to an obesogenic environment.

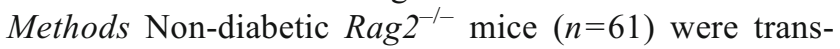
planted with human islets (400 islet equivalents [IEQ]) from six pancreases: four non-diabetic and two with overt metabolic dysfunction (older, high $\mathrm{HbA}_{\mathrm{lc}}$ or history of diabetes).
\end{abstract}

Electronic supplementary material The online version of this article (doi:10.1007/s00125-012-2775-y) contains peer-reviewed but unedited supplementary material, which is available to authorised users.

S. Gargani $\cdot$ J. Thévenet $\cdot$ J. E. Yuan $\cdot$ B. Lefebvre $\cdot$ N. Delalleau $\cdot$

V. Gmyr · T. Hubert - A. Duhamel · F. Pattou - J. Kerr-Conte

Université Lille Nord de France,

Lille, France

S. Gargani $\cdot$ J. Thévenet $\cdot$ B. Lefebvre $\cdot$ N. Delalleau $\cdot$ V. Gmyr $\cdot$

T. Hubert $\cdot$ A. Duhamel $\cdot$ F. Pattou $\cdot$ J. Kerr-Conte

UDSL, IMPRT,

Lille, France

S. Gargani $\cdot$ J. Thévenet $\cdot$ N. Delalleau $\cdot$ V. Gmyr $\cdot$ T. Hubert $\cdot$

F. Pattou $\cdot$ J. Kerr-Conte $(\bowtie)$

Inserm U859 Biotherapies of Diabetes, Faculty of Medicine,

1 Place de Verdun,

F59045 Lille, France

e-mail: jkerr-conte@univ-lille2.fr

A. Duhamel $\cdot$ F. Pattou $\cdot$ J. Kerr-Conte

Centre Hospitalier Régional Universitaire de Lille,

Lille, France

A. Duhamel

CERIM, Faculty of Medicine,

Lille, France

Present address:

B. Lefebvre

Inserm U837, Alzheimer and Tauopathies,

Lille, France
Animals were fed for 12 weeks with a control or high-fat diet (HFD), and followed for weight, serum triacylglycerol, fasting blood glucose and human C-peptide. After the mice were killed, human grafts and the endogenous pancreas were analysed for endocrine volume, distribution of beta and alpha cells, and proliferation.

Results Transplanted mice on an HFD gained significantly more weight $(p<0.001)$ and had higher fasting glycaemia (2-12 weeks; $p=0.0002)$ and consistently higher fasting human C-peptide levels $(2-12$ weeks; $p=0.04)$ compared with those on the control diet. Histology demonstrated doubling of human islet graft volume at 12 weeks in animals on the HFD and increased beta cell volume $(p<0.001)$, but no change in alpha cell volume. Human islet function (hyperbolic product $\mathrm{HOMA} 2 \% \mathrm{BS}$ ) at 12 weeks was four times lower in HFD animals $(p<0.001$ vs controls) because of insufficient beta cell adaptation to decreased (70\%) sensitivity (HOMA\%S). Human islets obtained from donors with metabolic dysfunction failed to adapt to the HFD.

Conclusions/interpretation This longitudinal study provides direct evidence that human islets adapt both endocrine and beta cell mass, function and gene expression to obesity in vivo. The present model will facilitate the identification of mechanisms by which human islets adapt to obesity in vivo and the cell type(s) responsible, and factors predisposing human beta cells to decompensation.

Keywords Expansion · High-fat diet · Human beta cells . Human islets · Obesity · Transplantation

$\begin{array}{ll}\text { Abbreviations } \\ \text { HFD } & \text { High-fat diet } \\ \text { HOMA2\%B } & \text { HOMA index of beta cell function } \\ \text { HOMA2\%S } & \text { HOMA index of insulin sensitivity } \\ \text { IEQ } & \text { Islet equivalents }\end{array}$




\section{Introduction}

Decoding how diabetes and obesity are interrelated is a complex and interdisciplinary research endeavour $[1,2]$. Islet adaptation to obesity is well established in rodents, where islets compensate for insulin-resistant states by increasing beta cell mass and function to maintain normoglycaemia [3]. Major differences have, however, emerged in the field of beta cell biology between rodent and human cells, making work with human islets crucial [4]. Indeed, clinically, most obese individuals do not develop diabetes because islets compensate for insulin resistance $[5,6]$. Yet direct evidence that human islet mass adapts longitudinally to obesity in vivo is lacking and, moreover, little information is available on the mechanisms and cell type(s) involved. Current evidence for increased beta cell mass in obese humans (vs lean) is based entirely on postmortem histology [7]. Beta cell mass quantification in humans by in vivo imaging is nascent [8] and should one day enable longitudinal studies.

The aim of this study was to explore the longitudinal adaptation of human islets to an obesogenic environment. The transplantation of human islets into immune-compromised mice is a validated technique with which to study human beta cells in an in vivo environment [9] and when human C-peptide levels are measured, this technique provides a quantitative assessment of human islet function [10, 11]. In this study, we transplanted human islets into an immunodeficient mouse strain sensitive to high-fat diet (HFD)-induced obesity and show direct evidence that non-diabetic human islets adapt both endocrine and beta cell mass, function and gene expression to obesity in vivo. However, dysfunctional human islets showed arrested adaptation. This model may foster deciphering of the mechanisms by which human islets adapt to obesity and the cell type(s) that are responsible, and possibly identify key factors predisposing human beta cells to decompensation [1].

\section{Methods}

Human islets Pancreases were harvested from human braindeceased donors in agreement with French regulations and with our institutional ethics committee, and were processed for islet isolation as previously described [12]. Four of the pancreases were from non-diabetic donors and two were from donors with overt metabolic dysfunction (older, high $\mathrm{HbA}_{\mathrm{lc}}$ or a history of diabetes; see electronic supplementary material [ESM] Tables 1 and 2). All islets showed more than $90 \%$ viability after culture, but were quantitatively insufficient for clinical islet transplantation [13].

Animals, human islet transplantation and induction of obesity Male C57BL6 Rag $2^{-1-}$ immunodeficient mice of
8-9 weeks old ( $n=61$; courtesy of A. Bouloumié, Inserm U858, Toulouse, France [14], and RAGN12-M, Taconic, Germantown, NY, USA) were transplanted under the kidney capsule as previously described [10] with 400 human islet equivalents (IEQ). Mouse studies were performed in accordance with the guidelines from the local animal experimentation committee. As previously described, islets from each human donor were distributed symmetrically across experimental groups to control for potential differences in islet quality between the donors [15]. Eight to ten mice were transplanted per donor per condition. Animals were fed for 12 weeks with a control diet or HFD (NJ ref D12450B; Research Diets, New Brunswick, NJ, USA; ESM Table 3).

All mice were followed for 12 weeks for weight, serum triacylglycerol, $6 \mathrm{~h}$ fasting blood glucose and human $\mathrm{C}$-peptide. At the end of the study, mice were fasted for $6 \mathrm{~h}$ before receiving an oral bolus of glucose $(2 \mathrm{~g} / \mathrm{kg}$ body weight) diluted in saline buffer ( $0.9 \%$ sodium chloride). Tail blood was collected at 0,30 and 60 min after administration to evaluate human C-peptide levels (ultrasensitive human C-peptide kit; Mercodia, Uppsala, Sweden). Blood glucose was measured up to 180 min (Accu-Chek Go glucometer; Roche, Meylan, France). HOMA, calculated using fasting human C-peptide and blood glucose levels in mice (www.dtu.ox.ac.uk/homacalculator/index.php) [16], was used as an index of insulin sensitivity (HOMA2\%S), beta cell function (HOMA2\%B) and the hyperbolic product (HOMA $2 \% \mathrm{~S} \times \mathrm{HOMA} 2 \% \mathrm{~B} / 100$ ). Results of 12 clinicalgrade islet preparations (glycaemia, human C-peptide) transplanted into 20 immunodeficient mice, as previously described [10], were used to calculate theoretical HOMA2\%B and HOMA2\%S. Fat content was quantified by MRI using ten images/mouse before killing, as previously described [17].

Immunohistochemistry To assess proliferation, BrdU was injected $18 \mathrm{~h}$ before the mice were killed or administered for 7 days in the drinking water. Both the endogenous mouse pancreas and human grafts were fixed and embedded in paraffin for sectioning and analysis of endocrine mass, distribution of beta cells and proliferation. Endocrine mass of the endogenous pancreas was determined with Papanicolaou staining of sections [18]. For endocrine mass assessment in human islet grafts, the entire block was sectioned; one section every $50 \mu \mathrm{m}$ was immunolabelled with chromogranin A primary antibody (M0869, 1/100; Dako, Glostrup, Denmark) and revealed with Envision (K4003; Dako). Similarly, beta and alpha cell volumes were determined using Alexa 488-conjugated anti-human C-peptide (green) (Monsam, Uden, the Netherlands) or anti-glucagon (G2654; Sigma, SaintQuentin-Fallavier, France) using the Lightning-Link Conjugation Kit (Innova Biosciences, Cambridge, UK); nuclei were stained with DAPI (blue) (H-1200J; Vector Laboratories, Burlingame, CA, USA). 
Endocrine or beta cell proliferation in grafts was respectively determined with three-colour fluorescence staining, BrdUChromogranin A-DAPI or BrdU-insulin-DAPI, or Ki67replaced BrdU (MIB-1; M7240, 1/100; Dako). After antigen retrieval (microwave, citrate buffer $\mathrm{pH} 6$ ), tissues were incubated overnight at $4^{\circ} \mathrm{C}$ with primary rat anti-BrdU antibody (AbD, BU1/75, 1/100; Serotec, Paris, France) followed by goat anti-rat Alexa Fluor 594 antibody (A11007, 1/300; Invitrogen, St Aubin, France). Double labelling was performed with rabbit anti-chromogranin A (M0869, 1/100; Dako) or mouse anti-insulin/proinsulin (AbD; D6C4, 1/300; Serotec), followed by donkey anti-rabbit Alexa Fluor 488 antibody (A21206, 1/300; Invitrogen) or goat anti-mouse Alexa Fluor 488 (A11001, 1/300; Invitrogen). Double labelling for chromogranin A, the pan-endocrine marker, and cytokeratin 19, as described $[19,20]$ suggestive of endocrine neogenesis, was quantified as previously described (the number of CK19positive ductal cells co-stained with chromogranin A) [7].

Morphometric analysis To quantify the total islet surface of human graft sections and islet surface in mouse pancreases, slides were scanned with a Nikon Eclipse Ti microscope, and the fractional area was digitally quantified (Nis Elements AR 3.0) (Nikon, Champigny sur Marne, France). Planimetry, used for clinical tumour volume appreciation (surface in microns ${ }^{2} \times$ distance in microns between paraffin sections), was used to determine endocrine, beta and alpha volumes $\left(\mu \mathrm{m}^{3}\right.$ or $\mathrm{mm}^{3}$ ) of entire human islet grafts. Leica DM-R microscope and ImageJ (National Institutes of Health, Bethesda, MD, USA) were used for the quantitative analysis.

RNA tissue extraction and Q-PCR RNA was isolated from snap-frozen human islet grafts $(n=14)$ immediately after killing using the RNeasy Micro Kit (Qiagen, Paris, France). RNA quantification was performed with the NanoDrop spectrophotometer (ND-1000; Thermo Scientific, Wilmington, NC, USA) and reverse transcription of $0.5 \mu \mathrm{g} / \mu \mathrm{l}$ RNA was performed with the High Capacity cDNA Reverse Transcription Kit (Applied Biosystems, Paris, France). For small human samples, pre-amplification of the gene of interest was performed with the TaqMan PreAmp Kit (Applied Biosystems), followed by quantitative PCR analysis (SYBR Green; Applied Biosystems). The INS primers (forward: 5'GCAACTAGACGCAGCCCGCA'3; reverse: 5'GGCTTTATTCCATCTCTCTCGGTGC' 3 ) were designed on the basis of sequence alignments of the INS region of human species and unrelated to the mouse; $\beta$-actin was the reference gene. Only one amplification product for $I N S\left(86^{\circ} \mathrm{C}\right)$ and for actin $\left(82^{\circ} \mathrm{C}\right)$ was observed in human islets grafts and none for genomic DNA from the mouse kidney.

Statistical analysis For each variable, the change over time ( 2 and 12 weeks; denoted $2-12$ weeks) as well as the difference at any given time was compared according to the two groups (control vs HFD) using analysis of variance based on a linear mixed model in order to adjust for the donor effect. The fixed effect was the group and the random effect was the donor. The same procedure was used to compare the histological data at 12 weeks according to the two groups. The analysis of repeated measurements for weight was also performed using the linear mixed model. In this analysis, the effect 'donor' was considered as a random effect and the correlations between the repeated measurements were modelled by using a first autoregressive covariance model. Our choice of this model was based on Akaike's information criterion. The validity of the linear mixed model was checked using the analysis of the residuals; the normality of residuals was tested with the ShapiroWilk test and the outliers were identified using box plots. The influence of each individual on the results was investigated using the Cook distance. If necessary, the stability of results after removing outliers or influential individuals was checked. Data were plotted as mean \pm SEM.

Statistical analyses were performed using StatView (SAS Institute, Cary, NC, USA) and SAS (SAS Institute Inc., Cary, NC, USA). $p$ values $<0.05$ were considered statistically significant.

\section{Results}

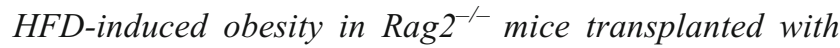
human islets A preliminary study using young (4-weekold) or adult male immunodeficient C57BL6 $\mathrm{Rag}^{-1-}$ mice $(n=14)$ confirmed that adults, as suggested $[14,21]$, gain weight on an HFD similar to male C57BL6 wild-type animals after 12 weeks ( $p=0.110$, results not shown). All subsequent experiments used adult male $\operatorname{Rag}^{2^{-/-}}$mice with a human islet graft.

Even with a human islet graft, $R a g 2^{-/-}$mice on a HFD gained considerable weight (Fig. 1a) as compared with mice on the control diet $(p<0.0001)$. This corresponded to higher BMI, serum triacylglycerol levels (Fig. 1b) and fat indices (ESM Table 4), including abdominal fat measured with MRI (Fig. 1c: HFD $52.27 \pm 4.39 \%$ vs controls $25.7 \pm 0.604 \%, p<$ 0.05 ) in adult $\operatorname{Rag}^{2^{-1-}}$ HFD mice vs controls.

Confirmation of adaptation of endogenous pancreas Morphometric analysis of the endogenous mouse pancreas confirmed that total islet area was doubled in HFD mice. There was no difference in the number of islets (per $\mathrm{mm}^{2}$ tissue surface) between the two groups (ESM Table 4), but the islets were larger and the proportion of beta cells higher in the pancreases of mice on the HFD at 12 weeks. Total pancreatic and endocrine cell proliferation was elevated in the endogenous pancreases of HFD mice. 
a
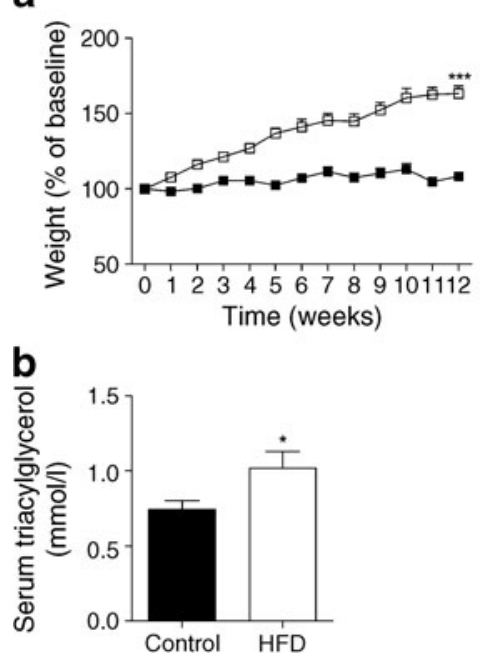

Fig. 1 (a) Weight gain ( $\%$ of baseline) over 12 weeks in animals grafted with human islets from four donors. White squares, HFD grafts $(n=23)$; black squares, controls $(n=22)$ (linear mixed model, see Methods, $* * * p<0.001$ ). (b) Serum triacylglycerol levels at 12 weeks $(* p<0.05$ vs controls). (c) MRI was used to quantify (10 sections/ mouse) abdominal fat content (expressed as a percentage \pm SEM) before the mice were killed (HFD 52.27 $\pm 4.39 \%$ vs controls $25.7 \pm$ $0.604 \%, * p<0.05$, fat appears in white)

Histological assessment at 12 weeks of the human grafts in animals on control or HFD Morphometric analysis with chromogranin A (Fig. 2a, 196-210 sections/group; see Methods) confirmed the more than doubling of human endocrine graft volume (HFD $0.094 \pm 0.017$ vs controls 0.042 $\pm 0.008 \mathrm{~mm}^{3} ; p=0.0006$ ) (Fig. 2b). To determine if this was due to endocrine cell hypertrophy of human islets as proposed [22] in HFD animals, individual cell surfaces were measured; no difference was observed (HFD $138 \pm 5.9 \mu \mathrm{m}^{2}$ vs controls $\left.135.3 \pm 4.6 \mu^{2} ; p=0.7\right)$. Indeed a 2.6 -fold increase in beta cell volume (Fig. 2c; HFD $0.071 \pm$ $0.0021 \mathrm{~mm}^{3}$ vs controls $0.027 \pm 0.001 \mathrm{~mm}^{3} ; p=0.0006$ ) was observed at 12 weeks (Fig. 2d), yet alpha cell volume was identical between the groups (Fig. 2e; HFD $0.011 \pm 0.002 \mathrm{~mm}^{3}$ vs controls $\left.0.009 \pm 0.002 \mathrm{~mm}^{3} ; p=0.0735\right)$. It is worth noting that human beta cells represented $64 \pm 2.0 \%$ and $75 \pm 1.86 \%$ of the total endocrine volume at 12 weeks in control and HFD mice, respectively $(p<0.05)$, whereas alpha cells made up a $18.08 \pm 1.49 \%$ in control animals and a statistically significantly smaller proportion in HFD animals $(12.45 \pm 1.69 \%, p<$ $0.05)$. Relative expression of human INS mRNA was 3.4-fold higher in human islets grafted in HFD mice (Fig. 3).

Total proliferation in human grafts, assessed at 12 weeks in all donors after overnight incubation with BrdU, was four times greater in HFD animals (BrdU-positive nuclei/total nuclei in graft: HFD $1.027 \pm 0.259 \%$ vs controls $0.259 \pm$ $0.157 \% ; p=0.03$ ). When BrdU was administered 1 day prior to mouse killing at 12 weeks, endocrine islet cell proliferation (double chromogranin A/BrdU cells) (Fig. 4a) was low

\section{a}

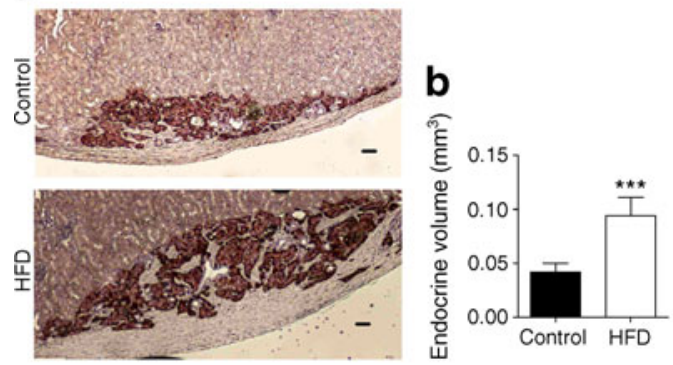

C
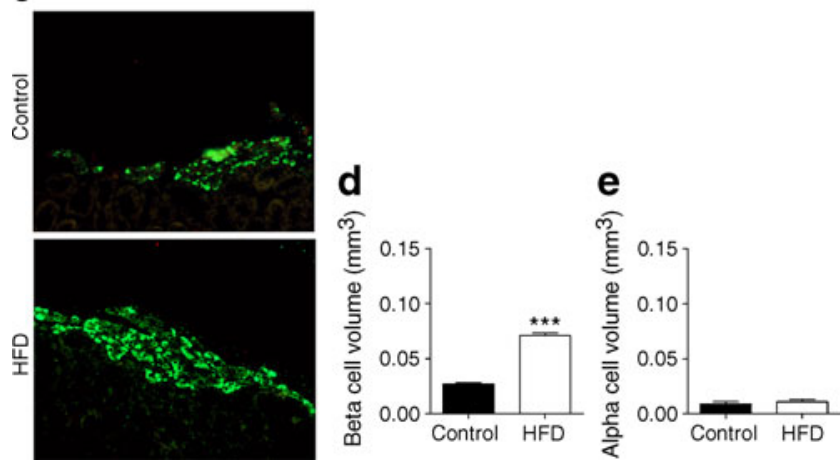

Fig. 2 (a) Representative sections of paraffin-embedded human grafts stained with the endocrine marker chromogranin A (DAB, brown) in HFD and control-diet mice (scale bar, $50 \mu \mathrm{m}$ ). (b) Morphometric analysis for total endocrine volume (chromogranin A) (HFD $[n=6]$ vs control [ $n=7],>196$ sections/group; linear mixed model, see Methods, $* * * p<0.001)$. (c) Sections of paraffin-embedded human grafts stained with the beta cell marker C-peptide (green). (d) Morphometric analysis for beta cell volume (HFD [ $n=6]$ vs control $[n=7], * * * p<0.001$ ). (e) Morphometric analysis for alpha cell volume (HFD $[n=6]$ vs control $[n=7])$

in all animals at 12 weeks (Fig. 4b), as was beta cell proliferation (double insulin/BrdU-positive cells; 1/1,500 cells; $p=\mathrm{NS}$ ). Anti-human anti-Ki67 antibody (Fig. 4c) showed that general proliferation in human grafts was higher in HFD mice (HFD $0.467 \pm 0.082 \%$ vs controls $0.214 \pm 0.054 \%, p=0.03$ ) and endocrine cell proliferation (double chromogranin $\mathrm{A} / \mathrm{Ki} 67$ ) was higher, albeit insignificantly in HFD animals (HFD $0.254 \pm 0.065 \%$ vs controls $0.119 \pm 0.034 \% ; p=0.08$ ) (Fig. 4d). Beta cell proliferation

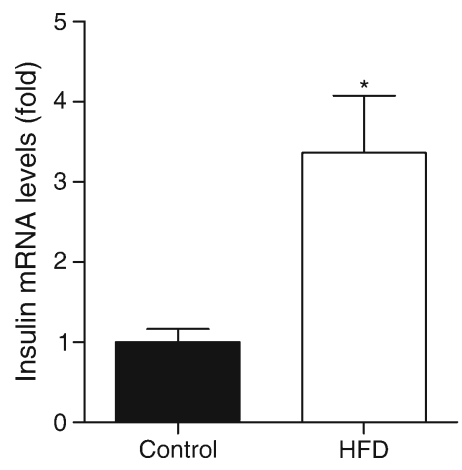

Fig. 3 Expression of the human INS gene in grafts (HFD [ $n=7]$ vs control $[n=7] ;{ }^{*} p<0.05$ ) (see Methods) 
Fig. 4 (a) Proliferation was determined on sections of human grafts at 12 weeks following an overnight incubation with BrdU (nuclei in blue, BrdU in red, chromogranin A [chromoA] in green or merge). (b) BrdU/ chromogranin A endocrinepositive cell quantification in human islet grafts (HFD $[n=4]$ vs control $[n=5])$. (c) Proliferation with Ki67 in beta cells (image shows merge of insulin in red, Ki67 in green). Quantification of (d) Ki67/chromogranin A and (e) Ki67/insulinpositive cells in human islets grafts (HFD $[n=4]$ vs control $[n=5]$; linear mixed model, see Methods, $p=0.08$ ) a

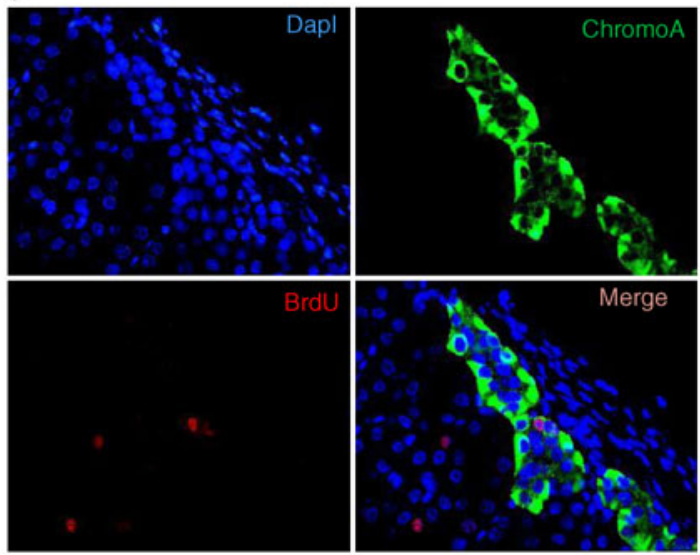

b

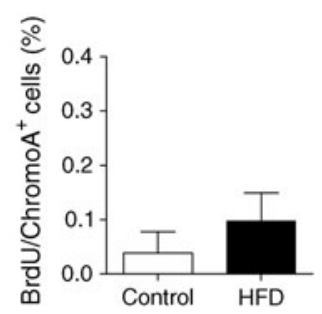

C

d

e

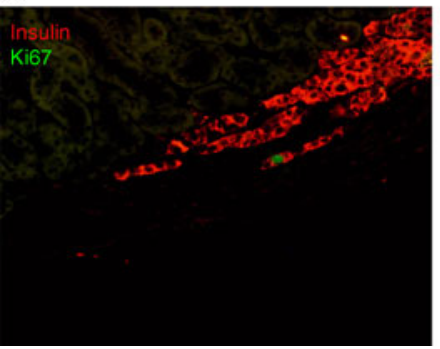

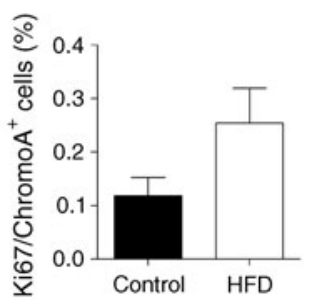

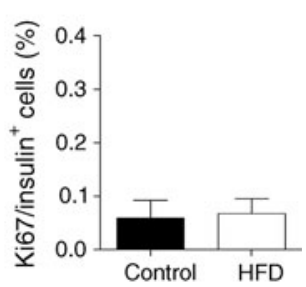

(double Ki67/insulin-positive) at 12 weeks showed no statistically significant difference between groups (HFD $0.067 \pm$ $0.033 \%$ vs $0.058 \pm 0.028 \%$; $p=0.1$ ) (Fig. 4 e).

We subsequently performed a kinetic study in mice transplanted with human islets, where BrdU was administered in the drinking water for 7 days prior to killing at 4, 6, 8 or 12 weeks (ESM Fig. 1a). Cumulative 7 day chromogranin A-positive endocrine proliferation (not shown) appeared higher at 12 weeks in HFD animals vs controls in accordance with the 1 day BrdU labelling, but also at 6 weeks HFD (results not shown). We also readily detected (4\%) 7 day cumulative proliferation in C-peptide-positive human beta cells at 6 and 12 weeks. Double labelling for ductal (anti-cytokeratin 19) and a pan-endocrine marker (anti-chromogranin A) was maximum at 8 weeks HFD (vs controls), suggesting the potential contribution of endocrine neogenesis (ESM Fig. 1b). Although further confirmation is required, both proliferation and neogenesis appear to potentially contribute to the expanded human islet mass in animals on an HFD.

Assessment of human islet graft function Fasting glycaemia was higher in HFD mice compared with controls at $2-12$ weeks $(p=0.0002)$ (Fig. 5a). It should be noted that the fasting glycaemia of $\mathrm{Rag}^{-/-}$mice $(n=11)$ with no islet graft was $6.83 \pm 0.28 \mathrm{mmol} / \mathrm{l}$. Compared with controls, HFD animals had a tendency towards higher plasma human C-peptide levels after 12 weeks compared with 2 weeks ( 2 weeks: controls $172.42 \pm 28.75$ pmol/1 vs HFD $479.54 \pm$ a

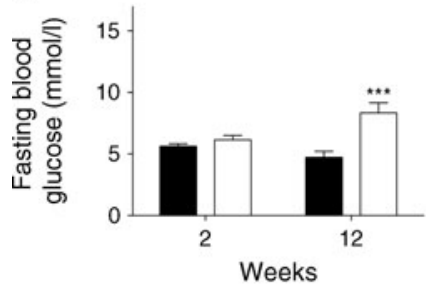

C

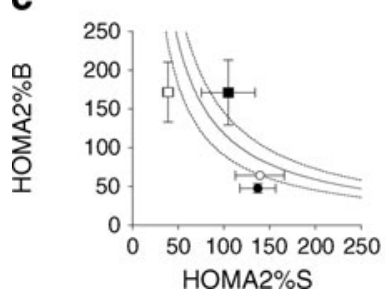

b

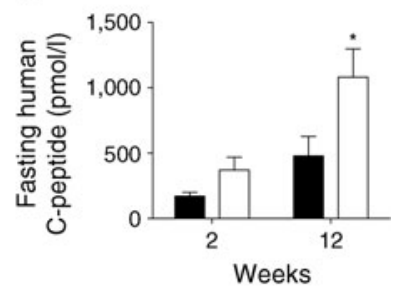

d

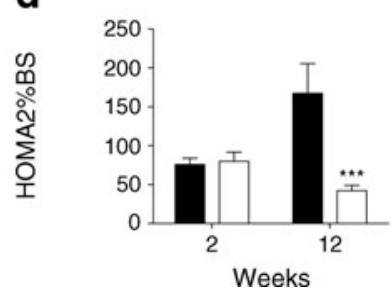

Fig. 5 (a) Fasting glycaemia (mmol/l) in animals with a human graft subsequently placed on an HFD (white bars) or control (black bars) diet $\left(2-12\right.$ weeks, linear mixed model, see Methods, $\left.{ }^{* * *} p<0.001\right)$. (b) Human C-peptide in tail blood plasma over the 12 week period in HFD (white bars; $n=20$ ) vs control diet (black bars; $n=19$ ) animals (2-12 weeks, linear mixed model, see Methods, ${ }^{*} p<0.05$ ). (c) Evolution of HOMA $2 \%$ B and HOMA $2 \% \mathrm{~S}$ in animals at 2 weeks (circles) or 12 weeks (squares) on an HFD (white) or control diet (black) (mean \pm SEM), plotted on hyperbolic curve (see Methods; the dotted lines represent the upper and lower 95\% CIs of the mean). (d) Hyperbolic product (HOMA2\%BS) in mice implanted for 12 weeks with nondiabetic human islets (HFD) [white bars] vs controls [black bars] at 12 weeks $(* * * p<0.001 ; * p<0.05$ controls, 2 vs 12 weeks) 
$148.84 \mathrm{pmol} / \mathrm{l} ; 12$ weeks: controls $370.89 \pm 97.91 \mathrm{pmol} / 1 \mathrm{vs}$ HFD 1,081.41 \pm 216.32 ; 2-12 weeks, $p=0.04$ ) (Fig. 5b). Results for individual human islet donors are depicted in ESM Fig. 2. Human C-peptide expressed as a function of glycaemia was also higher at 12 weeks (vs controls).

We used a surrogate HOMA2 based on fasting glycaemia and human C-peptide levels to longitudinally determine changes in human islet graft function in animals at 2 and 12 weeks. Figure 5c shows HOMA2\%B and HOMA2\%S for animals with respect to hyperbolic product value (HOMA2\%B $\times$ HOMA2\% $\% / 100$ ), calculated as described in the Methods (the dotted lines represent the upper and lower $95 \%$ confidence intervals of the mean). No difference was observed at 2 weeks between the two groups. An increase in HOMA $2 \%$ B was observed with time in both control and HFD animals (respectively, 2-12 weeks, $p=0.0075$ and $p=$ 0.06). Control animals showed no change in HOMA $2 \% \mathrm{~S}$ over time $(p=0.412)$; however, HFD animals showed a deterioration in HOMA $2 \% \mathrm{~S}(>70 \%$ at 12 week vs 2 weeks, $p=0.03$ ) corresponding to a 1.7 -fold increase in insulin resistance $(\mathrm{HOMA}-\mathrm{IR}=1 / \mathrm{HOMA}-\mathrm{S} \times 100$ ) over time and compared with control animals at 12 weeks. Increased peripheral insulin resistance was also confirmed in insulin tolerance tests (not shown). As a result, human islet function (hyperbolic product HOMA2\%BS) showed an improvement over time (2-12 weeks, $p=0.02)$ in control animals that was not observed in HFD animals at 12 weeks (Fig. 5d); HOMA2\%BS at 12 weeks was four times lower in HFD animals (vs controls, $p<0.001)$ due to insufficient beta cell adaptation to decreased $(70 \%)$ sensitivity $(\mathrm{HOMA} \% \mathrm{~S})$. An OGTT $(n=18$ mice) at 12 weeks (Fig. 6a) showed slightly abnormal glucose tolerance in HFD animals (vs $\mathrm{AUC}_{\text {glucose }}$ in control group, $p=$ 0.001); basal human C-peptide secretion and 60 min post glucose stimulation were higher in islets transplanted in HFD animals (vs controls, $p=0.06$; Fig. 6b), but only control islets were stimulated by glucose.

To further test the model, we evaluated the adaptation of islets isolated from two donors with overt disturbances in glucose metabolism (older donors, high $\mathrm{HbA}_{\mathrm{lc}}$ or a history
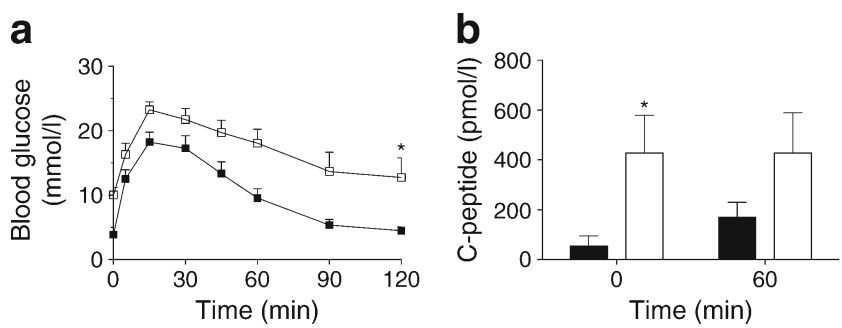

Fig. 6 (a) Representative blood glucose levels during an OGTT 12 weeks after human islet transplantation, HFD (white squares, $n=$ 9) vs control diet (black squares, $n=9)(5-120 \mathrm{~min}, * p<0.05)$. (b) Human C-peptide secretion at 0 and $60 \mathrm{~min}$ in islets transplanted in HFD animals (white bars) compared with controls (black bars) (HFD vs controls at $0 \mathrm{~min},{ }^{*} p<0.05$, and at $60 \mathrm{~min}, p=0.06$ ) of diabetes; ESM Table 2). As described in the Methods, islets from each donor were distributed symmetrically across the experimental groups. Despite identical weight gain, higher blood glucose levels were observed earlier in HFD animals grafted with diabetic islets. No functional adaptation to HFD was observed (HOMA-B and C-peptide/glycaemia levels were lower in the HFD group vs controls), which was confirmed by low HOMA $2 \%$ BS values at 12 weeks. Morphometric analysis showed no adaptation of endocrine volume to the HFD compared with control nondiabetic donors, and beta cells only accounted for approximately $10 \%$ of diabetic islets (\% C-peptide surface/chromogranin A surface) compared with $64 \pm 2.0 \%$ (control) to $75 \pm 1.86 \%$ (HFD) in non-diabetic donor islets (ESM Fig. 3). No proliferation could be detected.

\section{Discussion}

In this study, we have explored the longitudinal adaptation of human islets to an obesogenic environment and showed direct evidence that non-diabetic human islets adapt both endocrine and beta cell mass, function and gene expression to obesity in vivo. In fact, Tyrberg et al were the first to show the proof of concept that human islets xenotransplanted into obese hyperglycaemic-hyperinsulinaemic ob/ob mice could adapt to murine obesity [23] but the mice required immunosuppression, which attenuates islet compensation [24], limiting graft follow-up to 2 weeks. After first testing C57BL6 male $n u / n u$ mice that do not gain weight on a 12 week HFD (results not shown), adult male $\mathrm{Rag}^{-1-}$ animals (lymphocyte $\mathrm{B}$ and $\mathrm{T}$ deficient) were selected after a pilot study, to address the question: can human islets adapt to an obesogenic environment in mice? In this particular study, we first purposely chose young donors since they are most likely to adapt. Second, we chose not to render the animals diabetic and to use a mini-human islet graft - 400 IEQ vs the 2,000 IEQ classically used to cure diabetes, since this enables one to follow the adaptation to obesity in both the endogenous murine islets as well as the human cells. In fact, a mini-graft of $<500$ islets was chosen since higher numbers of islets $(1,000,2,000$ and 3,000 IEQ) lead to an atrophy of endogenous islets (J. KerrConte, unpublished observation) in accordance with previous publications $[25,26]$.

Concomitant with the increased weight gain, doubling of abdominal fat, increased serum triacylglycerol and reduced insulin sensitivity (HOMA-S) in 12 week HFD animals (vs controls), we report that human islet grafts (400 islets) showed functional compensation, measured as a more than doubling of fasting human C-peptide in mouse serum (vs controls), and histological adaptation of islet endocrine mass including increased beta cells. Increased human islet C-peptide secretion may be the simple result of islet 
adaptation to hyperglycaemia [27] yet, in this study, increased blood glucose levels were only observed at 12 weeks; it should be noted that glycaemia in HFD animals was lower than in C57BL6 wild-type or Rag2 ${ }^{-/}$animals on an HFD (results not shown). This has been previously attributed to the human set point $(1.0 \mathrm{~g} / \mathrm{l})$ of transplanted cells compared with the mouse set point (1.5$2.0 \mathrm{~g} / \mathrm{l}$ ), as previously described for human embryonic stemcell-derived islets grafted into non-diabetic immunodeficient mice [11]. In addition, increased C-peptide/glycaemia was also observed over time in mouse fed an HFD (vs controls at 12 weeks).

The doubling of human endocrine islet volume and increased percentage of beta cells (\%beta/endocrine cells) paralleled the adaptation of the murine endogenous pancreas. At 12 weeks, total proliferation was higher in both the endogenous pancreas and the human graft in HFD animals. Human endocrine islet cell proliferation at 12 weeks, determined with Ki67, was higher in HFD grafts (vs controls, albeit $p=0.08$ ) but beta cell proliferation was extremely rare. In initial studies, BrdU was administered the day before killing and not for 4 [27] to 7 days or 1 month, as recently published [28], which might be responsible for the extremely low beta cell proliferation seen in our study. Ki67/chromogranin A-positive cells exhibited twofold greater proliferation than BrdU/chromogranin A-positive cells, but beta cell proliferation remained rare to absent.

In a subsequent kinetic study (ESM Fig. 1), BrdU was administered for 7 days in the drinking water prior to killing. Cumulative 7 day endocrine proliferation and C-peptide beta cell proliferation had a tendency to be higher at 6 and 12 weeks in HFD animals vs controls. Chromogranin A-positive ductal cells, suggestive of neogenesis, peaked at 8 weeks in HFD grafts. It should be noted that we purposely double-labelled with the pan-endocrine marker chromogranin A and cytokeratin 19, and not insulin/cytokeratin 19, in order to identify all neoformed endocrine cells as previously described $[19,20]$. Further kinetic studies are required, yet it would appear that both proliferation and neogenesis can potentially contribute to the expanded islet mass. Future kinetic studies will combine permanent tagging of human beta cells (or alpha cells) prior to transplantation, as previously described [29], to bring definitive proof of the cell origin responsible for the doubling of the endocrine mass.

In this study, we used the fasting state HOMA of beta cell function (HOMA2) in the mouse as a surrogate to follow modifications in islet function in vivo. Identical at 2 weeks, marked increases were observed in HOMA $2 \% \mathrm{~B}$ in both control and HFD animals with time. Only HFD led to a marked decrease in insulin sensitivity (HOMA-S). Classically, a reduction in insulin action in non-diabetic individuals is accompanied by compensatory increases in beta cell secretion
(HOMA2\%B) [30], where HOMA2\%BS thus remains unaltered. If islets are defective, reduced HOMA $2 \% \mathrm{BS}$ values are observed. In this study the hyperbolic product (HOMA2\%BS), which was identical in the two groups at 2 weeks post-transplantation, improved significantly in control animals over time (2 vs 12 weeks), suggesting a hyperfunctional state of the animals that had both a small ectopic human graft (400 IEQ) and a persisting endogenous pancreas. HOMA2\%BS did not increase in HFD animals between 2 and 12 weeks, and was four times lower than HOMA2\%BS in control animals at 12 weeks. This suggests that although human islets adjust to increased insulin resistance by increasing human C-peptide secretion, normal human islets in an obese environment are defective. This was confirmed by OGTT results, which were also abnormal at 6 and 12 weeks in HFD animals (vs controls).

One limitation of our study is the use of HOMA in our combined human-murine model. Exploitation of this clinical tool in the rodent has been described [31] but remains controversial [16, 32]. Use of HOMA in this study is interesting and specific (human C-peptide), but may be the subject of debate since fasting glycaemia in this model can be regulated by both the human graft and the endogenous pancreas still in place, which also adapts as evidenced by a doubling of the murine islet mass on an HFD. Nevertheless, in our study this variable mainly allowed us to compare the different groups, all of which had a human islet graft from the same donors and endogenous pancreas.

To further validate the model, we had the opportunity to study the adaptation capacity of human islets from two dysfunctional human donors. Despite similar weight gain, animals had higher fasting glycaemia and showed no functional (human C-peptide) or histological adaptation of endocrine graft volume at 12 weeks. The hyperbolic plot, as well as HOMA2\%BS, illustrates the lack of adaptation. Human islet grafts from dysfunctional donors were made up of only $10-20 \%$ beta cells compared with $75 \%$ in normal human islets. Part of the incapacity of dysfunctional islets to adapt may be linked to the age of the donors ( $>60$ years), as has been suggested [28,33], although other authors have incriminated BMI [27].

In conclusion, we exploited an immunodeficient mouse strain to determine the influence of an obesogenic environment on human islets, and show for the first time direct evidence that non-diabetic human islets adapt both endocrine and beta cell mass, function and gene expression to murine obesity in vivo. This novel model allows, for the first time, longitudinal studies of human islet adaptation to an obese murine environment and may be instrumental in deciphering pathways involved in human beta cell expansion, as well as in helping to identify factors predisposing human beta cells to undergo decompensation [1]. In 
addition, the concomitant presence of a mini-human islet graft and the endogenous murine pancreas, both of which doubled in islet/endocrine surface, will allow scientists to study in parallel alterations in murine and human cell expansion at the same time in response to obesity. This will be key to identifying major differences between human and murine pancreatic cells.

Acknowledgements The authors would like to thank the Agence de la Biomedicine and R. Ezzouaoui and V. Raverdy (Centre Hospitalier Régional Universitaire de Lille, Lille, France) for pancreas coordination, and S. Belaich, I. Aluka, E. Moerman, B. Lukowiak and our pancreas harvesting team for their technical contribution (Inserm U859 Biotherapies of Diabetes, Faculty of Medicine, Lille, France). We also thank A. Bouloumié (Inserm U858, Toulouse, France) for mice, and M.H. Gevaert and R.M. Siminski (Laboratory of Histology, Faculty of Medecine, Lille, France) for their technical expertise.

Funding $\mathrm{S}$. Gargani is the recipient of a 3 year $\mathrm{PhD}$ stipend from Conseil Regional Nord Pas de Calais/University Hospital of Lille. This work was supported in part by grants from Conseil Regional Nord Pas de Calais to the European Genomics Institute of Diabetes (EGID), Contrat Plan Etat Region, Inserm, European Consortium for Islet Transplantation funded by the Juvenile Diabetes Research Foundation International, Chicago Diabetes Project (J. Oberholzer), Agence de la Biomedecine and core facilities including the Biotherapies Platform provided by or funded by IFR 114 Institut de Medecine Predictive et de Recherche Therapeutique, and the University Hospital of Lille.

Duality of interest The authors declare that there is no duality of interest associated with this manuscript.

Contribution statement JKC, FP, TH, JT and SG conceived and designed the experiments. JT, JEY, SG, VG, ND and JKC acquired data. SG, JKC, JT, JEY, ND, VG and FP analysed the data. JT, SG, $\mathrm{JKC}, \mathrm{FP}$ and JEY wrote the paper. AD performed statistical analysis, interpretation and writing of the Methods section. BL was involved in the conception, interpretation and drafting of the molecular biology work. JKC, JT, ND, VG, SG, TH, JKC and FP wrote the Methods, and revised the paper. All authors discussed and agreed on the results. JKC supervised the project. All authors gave final approval.

Open Access This article is distributed under the terms of the Creative Commons Attribution License which permits any use, distribution, and reproduction in any medium, provided the original author(s) and the source are credited.

\section{References}

1. Eckel RH, Kahn SE, Ferrannini E et al (2011) Obesity and type 2 diabetes: what can be unified and what needs to be individualized? J Clin Endocrinol Metab 96:1654-1663

2. Prentki M, Nolan CJ (2006) Islet beta cell failure in type 2 diabetes. J Clin Invest 116:1802-1812

3. Sachdeva MM, Stoffers DA (2009) Minireview: meeting the demand for insulin: molecular mechanisms of adaptive postnatal beta-cell mass expansion. Mol Endocrinol 23:747-758

4. Parnaud G, Bosco D, Berney T et al (2008) Proliferation of sorted human and rat beta cells. Diabetologia 51:91-100
5. Kahn SE, Hull RL, Utzschneider KM (2006) Mechanisms linking obesity to insulin resistance and type 2 diabetes. Nature 444:840 846

6. Lee YC, Nielsen JH (2009) Regulation of beta cell replication. Mol Cell Endocrinol 297:18-27

7. Butler AE, Janson J, Bonner-Weir S, Ritzel R, Rizza RA, Butler PC (2003) Beta-cell deficit and increased beta-cell apoptosis in humans with type 2 diabetes. Diabetes 52:102-110

8. Pattou F, Kerr-Conte J, Wild D (2010) GLP-1-receptor scanning for imaging of human beta cells transplanted in muscle. N Engl $\mathrm{J}$ Med 363:1289-1290

9. Ricordi C, Scharp DW, Lacy PE (1988) Reversal of diabetes in nude mice after transplantation of fresh and 7-day-cultured (24 degrees C) human pancreatic islets. Transplantation 45:994-996

10. Caiazzo R, Gmyr V, Kremer B et al (2008) Quantitative in vivo islet potency assay in normoglycemic nude mice correlates with primary graft function after clinical transplantation. Transplantation 86:360-363

11. Kroon E, Martinson LA, Kadoya K et al (2008) Pancreatic endoderm derived from human embryonic stem cells generates glucoseresponsive insulin-secreting cells in vivo. Nat Biotechnol 26:443452

12. Vantyghem MC, Kerr-Conte J, Arnalsteen L et al (2009) Primary graft function, metabolic control, and graft survival after islet transplantation. Diabetes Care 32:1473-1478

13. Kerr-Conte J, Vandewalle B, Moerman E et al (2010) Upgrading pretransplant human islet culture technology requires human serum combined with media renewal. Transplantation 89:11541160

14. Duffaut C, Galitzky J, Lafontan M, Bouloumie A (2009) Unexpected trafficking of immune cells within the adipose tissue during the onset of obesity. Biochem Biophys Res Commun 384:482-485

15. McCall M, Pawlick R, Kin T, Shapiro AM (2012) Anakinra potentiates the protective effects of etanercept in transplantation of marginal mass human islets in immunodeficient mice. Am J Transplant 12:322-329

16. Lee S, Muniyappa R, Yan X et al (2008) Comparison between surrogate indexes of insulin sensitivity and resistance and hyperinsulinemic euglycemic clamp estimates in mice. Am J Physiol Endocrinol Metab 294:E261-E270

17. Papademetris X, Shkarin P, Staib LH, Behar KL (2005) Regional whole body fat quantification in mice. Inf Process Med Imaging 19:369-380

18. Lalloyer F, Vandewalle B, Percevault F et al (2006) Peroxisome proliferator-activated receptor alpha improves pancreatic adaptation to insulin resistance in obese mice and reduces lipotoxicity in human islets. Diabetes 55:1605-1613

19. Gmyr V, Kerr-Conte J, Belaich S et al (2000) Adult human cytokeratin 19-positive cells reexpress insulin promoter factor 1 in vitro: further evidence for pluripotent pancreatic stem cells in humans. Diabetes 49:1671-1680

20. Bouwens L (1998) Cytokeratins and cell differentiation in the pancreas. J Pathol 184:234-239

21. Winer S, Chan Y, Paltser G et al (2009) Normalization of obesityassociated insulin resistance through immunotherapy. Nat Med 15:921-929

22. Granot Z, Swisa A, Magenheim J et al (2009) LKB1 regulates pancreatic beta cell size, polarity, and function. Cell Metab 10:296-308

23. Tyrberg B, Ustinov J, Otonkoski T, Andersson A (2001) Stimulated endocrine cell proliferation and differentiation in transplanted human pancreatic islets: effects of the ob gene and compensatory growth of the implantation organ. Diabetes 50:301-307

24. Zahr E, Molano RD, Pileggi A et al (2007) Rapamycin impairs in vivo proliferation of islet beta-cells. Transplantation 84:15761583 
25. Flatt PR, Tan KS, Bailey CJ, Powell CJ, Swanston-Flatt SK, Marks $\mathrm{V}$ (1986) Effects of transplantation and resection of a radiationinduced rat insulinoma on glucose homeostasis and the endocrine pancreas. Br J Cancer 54:685-692

26. Blume N, Skouv J, Larsson LI, Holst JJ, Madsen OD (1995) Potent inhibitory effects of transplantable rat glucagonomas and insulinomas on the respective endogenous islet cells are associated with pancreatic apoptosis. J Clin Invest 96:2227-2235

27. Levitt HE, Cyphert TJ, Pascoe JL et al (2011) Glucose stimulates human beta cell replication in vivo in islets transplanted into NODsevere combined immunodeficiency (SCID) mice. Diabetologia 54:572-582

28. Tian L, Gao J, Weng G et al (2011) Comparison of exendin-4 on beta-cell replication in mouse and human islet grafts. Transpl Int $24: 856-864$
29. Russ HA, Bar Y, Ravassard P, Efrat S (2008) In vitro proliferation of cells derived from adult human beta-cells revealed by celllineage tracing. Diabetes 57:1575-1583

30. Bergman RN, Ader M, Huecking K, van Citters G (2002) Accurate assessment of beta-cell function: the hyperbolic correction. Diabetes 51(Suppl 1):S212-S220

31. Ng SF, Lin RC, Laybutt DR, Barres R, Owens JA, Morris MJ (2010) Chronic high-fat diet in fathers programs betacell dysfunction in female rat offspring. Nature 467:963966

32. Wallace TM, Levy JC, Matthews DR (2004) Use and abuse of HOMA modeling. Diabetes Care 27:1487-1495

33. Reers C, Erbel S, Esposito I et al (2009) Impaired islet turnover in human donor pancreata with aging. Eur J Endocrinol 160:185191 